\title{
Adenomatoid Odontogenic Tumor of Maxillary Sinus
}

\author{
${ }^{1}$ J agveer Singh Yadav, ${ }^{2}$ Satyawati Mohindra, ${ }^{3}$ Kirti G upta \\ ${ }^{1}$ Senior Resident, Department of Otolaryngology and Head and Neck Surgery, Postgraduate Institute of Medical \\ Education and Research, Chandigarh, India \\ ${ }^{2}$ Assistant Professor, Department of Otolaryngology and Head and Neck Surgery, Postgraduate Institute of Medical \\ Education and Research, Chandigarh, India \\ ${ }^{3}$ Assistant Professor, Department of Pathology, P ostgraduate Institute of Medical \\ Education and Research, Chandigarh, India
}

Correspondence: Satyawati Mohindra, Assistant Professor, Department of Otolaryngology and Head and Neck Surgery Postgraduate Institute of Medical Education and Research, Chandigarh-160012, India, P hone: +91-9914209765, Fax: +91-1722744401 e-mail: satyamohindra@gmail.com

\section{ABSTRACT}

Adenomatoid odontogenic tumor (AOT) is an uncommon tumor of odontogenic origin, constituting only $3 \%$ of all odontogenic tumors. AOT is characterized histologically by the formation of duct-like structures with amyloid-like deposits.

An 18-year-old male presented with right chek swelling. CT scan revealed well-defined expansile heterogeneous mass with central hypodensity. Patient underwent excision of the mass by Caldwell-luc approach and histopathologically the diagnosis adenomatoid odontogenic tumor was made. Since it is a benign tumor, the treatment should consist of enucleation and curettage.

Keywords: Adenomatoid odontogenic tumor, Caldwell-luc, Ameloblastoma.

\section{INTRODUCTION}

A denomatoid odontogenic tumor (AOT) is an uncommon tumor of odontogenic origin, constituting only $3 \%$ of all odontogenic tumors. ${ }^{1,2}$ It was first described by Stafne in $1948 .^{2}$ A OT is characterized histologically by the formation of duct-like structures with amyloid-like deposits. It was not until 1971 that the WHO adopted the term "adenomatoid odontogenic tumor" ${ }^{3}$ to describe this entity, as had been proposed by Philipsen and Birn. ${ }^{4}$ In the latest edition of WHO classification of odontogenic tumors in 2005, A OT was classified into the first group of tumors (odontogenic epithelium without ectomesenchyme) instead of the second group (odontogenic epithelium with ectomesenchyme) ${ }^{5}$ Because of the absence of ectomesenchyme in immunohistochemical staining and dysplastic dentin, A OT is now considered the result of a metaplastic process rather than epithelial-ectomesenchyme interaction. ${ }^{6}$ In this paper, we present a case of adenomatoid odontogenic tumor affecting the right maxillary region in a 16-year-old male. The authors also discuss clinical, radiographic, histopathologic and therapeutic features of the case.

\section{CASE REPORT}

A 18-year-old male was referred to the department of otorhinolaryngology and head and neck surgery, Postgraduate Institute of M edical Education and R esearch,
Chandigarh, India for evaluation of right side maxillary swelling. The medical history was insignificant. The patient was asymptomatic except facial swelling and was in good general health. Clinical examination showed facial asymmetry with a single $4 \times 4 \mathrm{~cm}$ swelling on right side of face.The swelling had ill-defined margins with normal overlying skin. On palpation, the swelling was bony hard and nontender. Intraoral examination disclosed a nontender expansion of the left maxilla, covered by normal mucosa. Teeth were nontender on percussion. CT scan showed well defined expansile heterogeneous tumor mass with central hypodensity occupying right maxillary sinus (Fig. 1). A provisional differential diagnosis of dentigerous cyst/ calcifying odontogenic cyst was considered.

Patient underwent excision via Caldwell-luc approach. The anterior wall of maxilla appeared to be thinned out but was intact. A reddish, bulky, cystic tumor became evident after removal of the anterior wall of maxilla. There was no apparent infiltration of the surrounding bones. The right upper canine tooth was completely embedded in the tumor. Cross examination of the specimen showed a single greybrown spherical mass measuring $5 \times 5 \mathrm{~cm}$. Cut section revealed a cyst with wall thickness of 0.1 to $0.5 \mathrm{~cm}$ with an impacted canine tooth. Postoperative period was uneventful. Patient does not have any signs of recurrence and is on regular follow-up. 
Histological examination revealed a partly cystic and partly solid tumor. The cyst was lined with hyperplastic amel oblastic epithelium with basal palisading (Fig 2A). At places, this lining was dispersed in form of nodular islands forming duct-like structures and glands (Figs $2 B$ and $C$ ). The glandular structures at higher magnification revealed tall columnar lining epithelium without significant nuclear atypia (Fig 2D). In addition, focally there was deposition of hyaline material in between the glandular structures. $\mathrm{N} 0$ mitotic figures or necrosis were identified. The features were of an adenomatoid odontogenic tumor (Figs 2A to D).

\section{DISCUSSION}

A OT had been initially described and classified as a variant of the ameloblastoma 7,8 named an adenoameloblastoma, adenoameloblastic odontoma, pseudoadenomatous ameloblastoma, cystic complex composite odontoma, unusual pleomorphic adenoma like tumor, ameoloblastic adenomatoid tumor, odontoameloblastic tumor, odontoameloblastic odontoma, tumor of enamel organ, ameloblastic epithelial tumor and tumor connected to development cysts. ${ }^{9}$ Pindborg named it adenomatoid odontogenic tumor (A OT), classifying it as an odontogenic epithelial tumor presenting inductive effect at the connective tissue. ${ }^{10}$

There are three clinicopathologic variants of AOT, namely intraosseous follicular, intraosseous extrafollicular and peripheral, all with identical histology. The follicular type is a central intraosseous lesion associated with an impacted tooth, while extrafollicular intraosseous A OT has no relation with an unerupted tooth. In spite of this, it is often located between, above or superimposed up on the

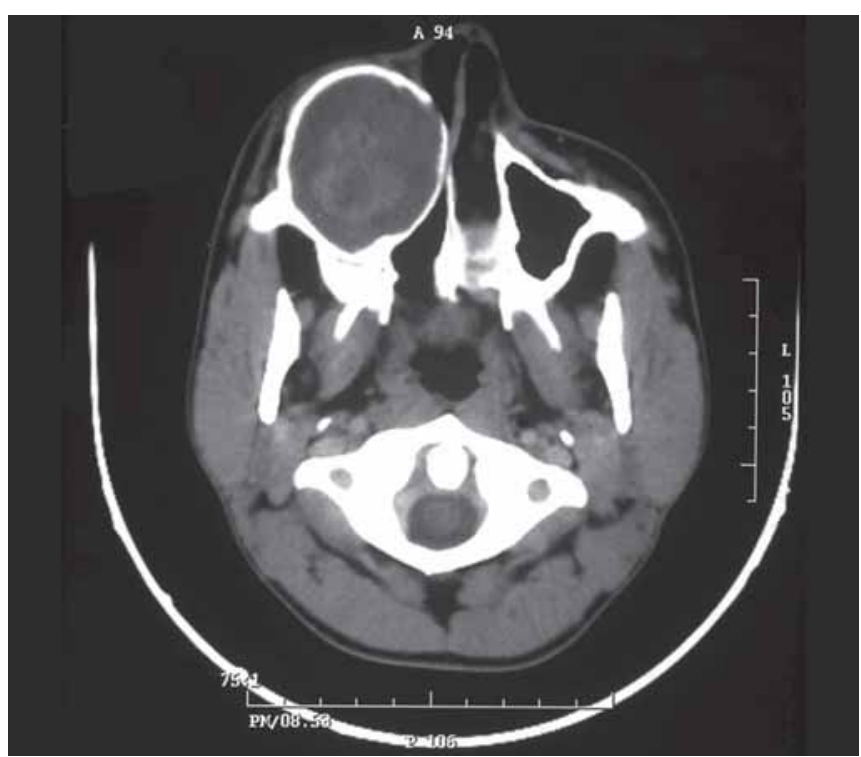

Fig. 1: Noncontrast computed tomograpy (axial cut) shows heterogenous soft tissue density with expansion of bony walls of right maxillary sinus roots of adjacent erupted teeth. The peripheral variant appears as a gingival fibroma or epulis attached to the labial gingival. ${ }^{10}$

The follicular and extrafollicular variants account for $96 \%$ of all A OT cases (of which $71 \%$ are follicular). Both intraosseous and extraosseous forms are distinguished. The subtyping of AOT is based on clinical and radiological findings. The follicular (intraosseous) type is by far the most frequent growth type of AOT. ${ }^{11}$ Follicular and extrafollicular variants together are more commonly found in the maxilla than in the mandible (2.1:1 ratio). M ore than two-thirds are diagnosed in the second decade, mostly in the 13 to 19 years age group. The female:male ratio is 1.9:1. ${ }^{10}$ Even higher ratios are found in A sian populations, the highest incidence being observed in Sri Lanka (3.2:1) ${ }^{12}$ and J apan (3:1). ${ }^{13}$

The tumor is usually associated with unerupted teeth, frequently canines or lateral incisors. Irregular root resorption is seldom reported. ${ }^{1,14}$ The patient we describe in this report also presented resorption of the upper left lateral incisor and first premolar, together with impaction of the canine. Radiologically, it should be differentiated from dentigerous cyst, which most frequently occurs as a pericoronal radiolucency in the jaws. Dentigerous cyst encloses only the coronal portion of the impacted tooth, whereas A OT shows radiolucency usually surrounding both the coronal and radicular aspects of the involved tooth. ${ }^{15}$

Common neoplastic causes, such as ameloblastoma, calcifying epithelial odontogenic tumor (CEOT), ameloblastic fibroma and ameloblastic fibro-odontoma are easily differentiated on histology. CEOT shows larger and more numerous calcifying spherules within eosinophilic cytoplasm of large cells along with smaller cells with hyperchromatic nuclei. A myloid-like eosinophilic material is also present. A meloblastoma has characteristic lining and arrangement with stellate reticulum besides usual location in mandible and posterior maxilla in contrast to AOT that is located in anterior maxilla. ${ }^{16} \mathrm{~A}$ reas of CEOT-like tissues have been described in classic A OT.

Immunohistochemical and ultrastructural findings have shown that the eosinophilic deposits (amyloid-like material) most probably represent some form of enamel matrix. The histogenesis of AOT is still uncertain, although recent findings strongly indicate that AOT is derived from a complex system of dental laminae or its remnants. ${ }^{17}$ It is often considered as a hamartomatous lesion rather than a true neoplasm. ${ }^{17}$

A review of the literature depicts a lesion in which conservative management produces a uniformly excellent outcome without recurrence. ${ }^{18,19}$ Since adenomatoid odontogenic tumor (AOT) is a benign tumor that presents with a nonaggressive biologic behavior, progressive growth, 


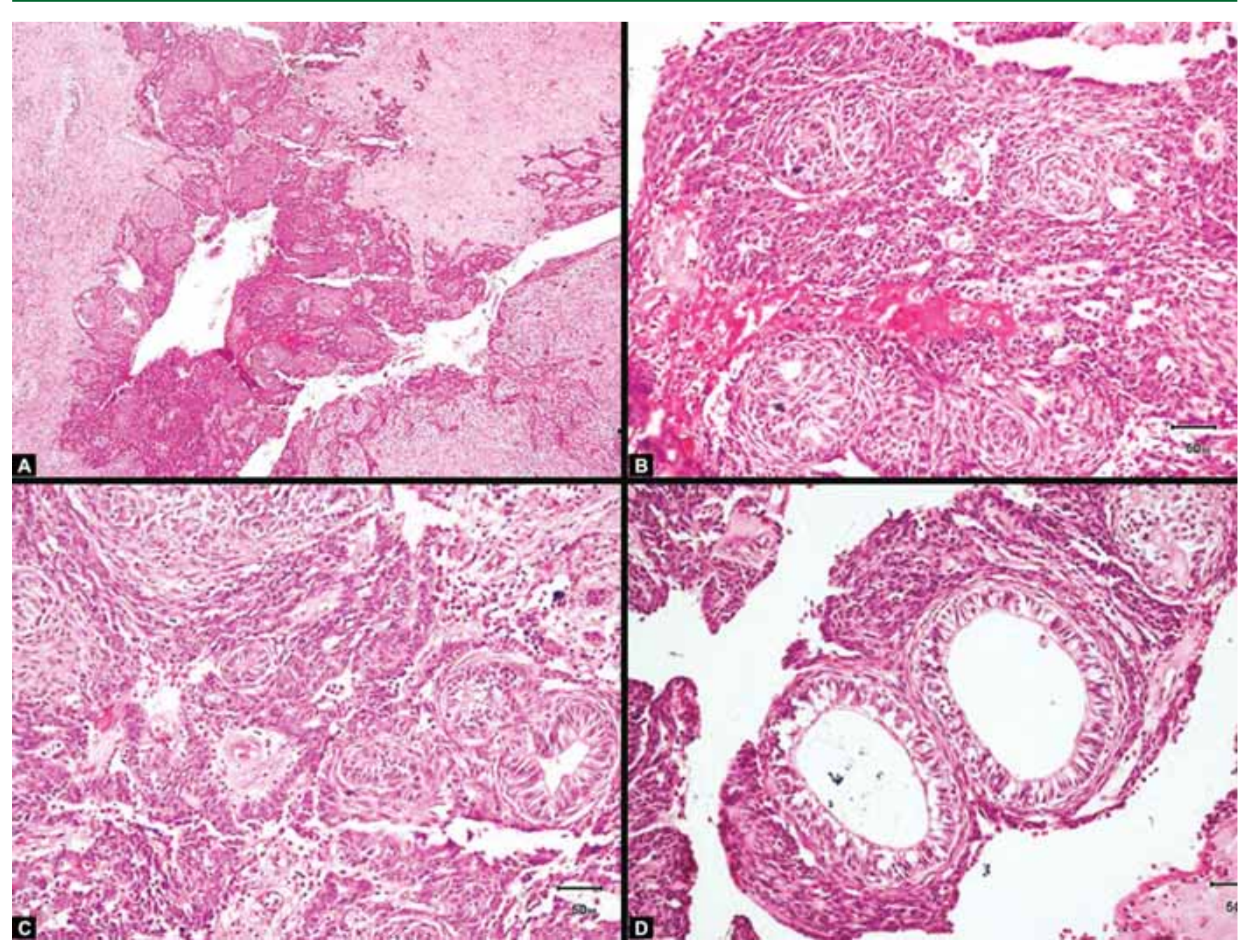

Figs 2A to D: (A) The cyst showing a lining composed of hyperplastic a meloblastic epithelium with basal palisading (original magnification, $H \& E \times 40)$, (B and C) Hyperplastic epithelium dispersed in form of nodular islands, center of which shows glands arranged in back to back configuration (original magnification, H\&E $\times 200$ ), (D) Glandular arrangement of cells with lining of tall columnar cells (original magnification, $H \& E \times 400)$

small frequency of recurrence, absence of invasion and the frequent presence of a connective tissue capsule, the treatment should consist of enucleation and curettage. ${ }^{20,21}$ Only a few reports deal with recurrent A OT and all are from Japan. ${ }^{18}$ The patient we described in this case report is healthy, without recurrence, and is under follow-up after local excision.

\section{CONCLUSION}

The large size of these lesions supports the classification of AOT as a benign neoplasm and not a hamartoma. By definition, a neoplasm has an unlimited growth potential, whereas hamartomas have a limited growth potential and differentiate into more mature tissue with time. It should be differentiated from ameloblastoma. A meloblastoma has characteristic lining and arrangement with stellate reticulum besides usual location in mandible and posterior maxilla in contrast to A OT that is located in anterior maxilla.

\section{REFERENCES}

1. Dayi E, Gurbuz G, Bilge OM, Ciftcioglu M A. A denomatoid odontogenic tumour (adenoameloblastoma). Case report and review of the literature. A ust DentJ 1997;42:315-18.

2. L ee JK, Lee K B, H wang B N . A denomatoid odontogenic tumor: A case report. J Oral M axill ofac Surg 2000;58:1161-64.

3. K ramer IRH, Pindborg JJ. Histological typing of odontogenic tumours (2nd ed). Springer, Berlin 1992.

4. Philipsen HP, Birn H. The adenomatoid odontogenic tumour. A meloblastic adenomatoid tumour or adenoameloblastoma. A cta Pathol Microbiol Scan 1969;75:375-98.

5. Jing $W, X$ uan $M$, L in Y, et al. Odontogenic tumours: A retrospective study of 1642 cases in a Chinese population. A $n$ International J ournal of Oral and M axillofacial Surgery 2007;36(1):20-25.

6. Evans BL, Carr RF, Phillipe LJ. A denomatoid ameloblastoma with dentinoid: A case report. Oral Surgery, Oral Medicine, Oral Pathology, Oral Radiology and Endodontology 2004;98(5): 583-88.

7. Dreiblat $\mathrm{H}$. U eber pseudoadenoma adamantinum. Inaugural dissertation, U niversität zu B erlin; 1927.

8. Ghosh LS. A damantinoma of upper jaw. Report of a case. Am J Pathol 1934;10:773-90. 
9. Philipsen HP, et al. A denomatoid odontogenic tumor: Biologic prole based on 499 cases. J Oral Pathol M ed 1991;20:149-58.

10. Pindborg JJ, Kramer IR. Tipos histológicos de tumors odontogênicos, quistos de los maxilares y lesions anes. Genebra, Organización M undial de la Salud, 1972, p. 28.

11. Philipsen HP, Reichart PA . A denomatoid odontogenic tumour: Facts and figures. Oral Oncol 1998;35:125-31.

12. Reichart PA, Philipsen HP. Odontogenic tumors and allied lesions. Quintessence Publ., London 2004; 105-15.

13. M endis BRRN, MacDonald DG. A denomatoid odontogenic tumour: A survey of 21 cases from Sri Lanka. Int J Oral Maxillofac Surg 1990;19:141-43.

14. Toida M, Hyodo I, Okuda T, Tatematsu N. A denomatoid odontogenic tumor: Report of two cases and survey of 126 cases in Japan. J Oral M axillofac Surg 1990;48:404-08.

15. Nomura M, Tanimoto K, Takata T, Shimosato T. M andibular adenomatoid odontogenic tumor with unusual clinicopathologic features. J Oral M axillofac Surg 1992;50:282-85.
16. Rosai J . M andibleand M axilla. In: A ckerman's Surgical Pathology (8th ed). Rosai J (Ed). St. L ouis: M osby; 1996;257-88.

17. Philipsen HP, Samman N, O rmiston IW, Wu PC, Reichart PA. $V$ ariants of the adenomatoid odontogenic tumor with a note on tumor origin. J Oral Pathol M ed 1992;21:348-52.

18. Gold L. Odontogenic tumors: Surgical pathology and management. In: Fonseca RJ (Ed). Oral and maxillofacial surgery. Philadel phia: W B Saunders; 2000;328-30.

19. Assael $L$, Peterson $L J$, et al (Eds). Surgical management of odontogenic cysts and tumors. In: Principles of oral and maxillofacial surgery, UK : Lippincott 1992;3:698-701.

20. Giansanti JS, Someren A, Waldron CA. Odontogenic adenomatoid tumor (adenoameloblastoma). Survey of 3 cases. Oral Surg Oral Med Oral Pathol 1970;30:69-88.

21. Sampaio RK, M oreira LC. Tumores odontogênicos. A spectos clýnìicos, radiográcos e tratamento. Rio de J aneiro, Rev- inter, 1992; 93-100. 